\title{
Prevalence and outcome of contrast-induced nephropathy in major trauma patients
}

\author{
Julian Alexander Kelemen ${ }^{1}$ - Alexander Kaserer ${ }^{2} \cdot$ Kai Oliver Jensen $^{1} \cdot$ Philipp Stein $^{2,4}$ • Burkhardt Seifert ${ }^{3,5}$. \\ Hans-Peter Simmen ${ }^{1} \cdot$ Donat R. Spahn ${ }^{2} \cdot$ Hans-Christoph Pape ${ }^{1} \cdot$ Valentin Neuhaus $^{1}$ (I)
}

Received: 27 April 2020 / Accepted: 4 September 2020 / Published online: 19 September 2020

(c) The Author(s) 2020

\begin{abstract}
Background Contrast-induced nephropathy (CIN) has been well investigated in patients undergoing coronary angiography, but not in trauma patients. The main aim of this study was to determine the prevalence and to investigate independent risk factors for the development of CIN.

Methods Between 2008 and 2014, all pre-hospital intubated major trauma patients with documented serum creatinine levels ( $\mathrm{SCr}$ ) undergoing a contrast-enhanced whole-body $\mathrm{CT}$ at admission were retrospectively analyzed. CIN was defined as a relative increase in $\mathrm{SCr}>25 \%$ over the baseline value or an absolute $\mathrm{SCr}$ increase of $>44 \mu \mathrm{mol} / \mathrm{l}$ within $72 \mathrm{~h}$. Univariate and multivariable regression analyses were performed to identify significant risk factors. A $p$ value of $<0.01$ was considered statistically significant and a $p$ value of $0.01-0.049$ suggested evidence.

Results Of 284 analyzed patients, $41(14 \%)$ met the criteria for CIN. There is suggestive evidence that age and lactate level influenced the development of CIN. Six patients (15\%) had hemodialysis in the CIN-group and eight (3.3\%) in the group without CIN. Complication and mortality rate was higher in patients with $\operatorname{CIN}(71 \%$ vs. $56 \%$ and $32 \%$ vs. $23 \%$, respectively). CIN was not an independent risk factor for complications or mortality while controlling for age, gender, injury severity score, and lactate level. The length of stay was not affected by CIN.

Conclusion CIN occurs frequently in trauma patients, but is not an independent risk factor for complications or mortality. Therefore, contrast enhanced whole-body CT can safely be performed in trauma patients.
\end{abstract}

Keywords Computed tomography $\cdot$ Contrast $\cdot$ Nephropathy $\cdot$ Trauma

Valentin Neuhaus

valentin.neuhaus@gmx.ch

Julian Alexander Kelemen

julianalexander.kelemen@gmail.com

Alexander Kaserer

alexander.kaserer@usz.ch

Kai Oliver Jensen

KaiOliver.Jensen@usz.ch

Philipp Stein

philipp.stein@ksw.ch

Burkhardt Seifert

seifert@ifspm.uzh.ch

Hans-Peter Simmen

HansPeter.Simmen@usz.ch

Donat R. Spahn

Donat.Spahn@usz.ch
Hans-Christoph Pape

hans-christoph.pape@usz.ch

1 Division of Trauma Surgery, Department of Surgery, University Hospital Zurich, University of Zurich, Raemistrasse. 100, 8091 Zurich, Switzerland

2 Institute of Anesthesiology, University and University Hospital Zurich, Raemistrasse 100, 8091 Zurich, Switzerland

3 Institute of Epidemiology, Biostatistics and Prevention, University of Zurich, Zurich, Switzerland

4 Institute of Anesthesiology, Cantonal Hospital Winterthur, Brauerstrasse 15, 8400 Winterthur, Switzerland

5 Department of Biostatistics at Epidemiology, Biostatistics and Prevention Institute, University of Zurich, Hirschengraben 84, 8001 Zurich, Switzerland 


\section{Abbreviations}

AKI Acute kidney injury

CIN Contrast-induced nephropathy

CPR Cardiopulmonary resuscitation

FFP Fresh frozen plasma

HES Hydroxyl ethyl starches

ISS Injury severity score

PCI Percutaneous coronary intervention

RBC Packed red blood cells

$\mathrm{SCr}$ Serum creatinine levels

\section{Background}

Contrast-induced nephropathy (CIN) is characterized as an acute renal injury after the administration of intravascular iodinated radio-contrast medium in the absence of any other etiology [1]. Although the histological morphology of CIN is well characterized, the pathophysiologic mechanism of renal injury is still not clear. Most likely a combination of reduced renal perfusion, a reduction in tubular flow and/ or a direct tubular toxicity of contrast agent could lead to a decrease in glomerular filtration rate $[1,2]$. CIN is commonly diagnosed by a $25 \%$ rise from baseline creatinine, or an absolute increase in creatinine of $\geq 44 \mu \mathrm{mol} / 124-48 \mathrm{~h}$ after administration of contrast [3-6].

The incidence of CIN varies from 0.6 to $2.3 \%$ in patients without any pre-existing renal impairment and rises up to $30 \%$ in the presence of individual risk factors [7]. Advanced age, diabetes mellitus, pre-existing renal impairment, periprocedural intravascular volume depletion, congestive heart failure or concomitant use of other nephrotoxic drugs have been shown to increase the risk to develop CIN [2, 3, $6,8,9]$. CIN is the third leading cause of hospital acquired acute renal failure and associated with a significant increase in mortality [2, 3]. Hence, several strategies (e.g., pre- and post-hydration, administration of $\mathrm{N}$-acetylcysteine) were proven to be more or less effective in the prevention of CIN [10], but are not feasible in every situation-especially in the treatment of severely injured patients. Further, the cause of creatinine increase in severely injured patients can be multifactorial (e.g., contrast-induced, hemorrhagic shock, blood transfusions, advanced age).

Whole-body computed tomography (CT) was shown to reduce mortality in polytraumatized patients, if integrated into early trauma care $[11,12]$. Therefore, contrast enhanced whole-body CT is more and more routinely performed for the initial evaluation of severely injured patients. Although crucial in the care of traumatized patients, only a few studies focus on the incidence, the impact and the clinical implications of CIN in those patients and the risk factors are discussed controversially [13-15]. Finigan et al. could not identify any risk factor for CIN in their work [14] and Kim et al. reported age $\geq 65$ years and ISS $\geq 25$ to be independently associated with acute renal injury, but not intravenous administered contrast medium [15]. The STARSurg Collaborative could not find any association between preoperative intravenous contrast administration and postoperative acute kidney injury after major gastrointestinal surgery [16].

To improve the understanding of CIN and its impact on severely injured patients, we conducted a retrospective analysis in our Level 1 trauma center. The main aim of this study was to determine prevalence of and to identify independent risk factors for CIN. Additionally, we assessed the clinical impact of CIN on the need of hemodialysis, complications rates, length of stay and mortality.

\section{Materials and methods}

This retrospective cohort study was approved by the local ethics committee (Kantonale Ethikkommission Zürich, Switzerland, KEK ZH No. 2011-0382) and adheres to the Strengthening the Reporting of Observational Studies in Epidemiology (STROBE) recommendations for cohort studies $[17,18]$.

\section{Study design and participants}

We included all adult, pre-hospital intubated trauma patients admitted to the emergency department of the University Hospital Zurich between October 2008 and December 2014 who received a contrast enhanced whole-body CT directly on arrival $(n=350)$. Patients with missing initial or followup (within $72 \mathrm{~h}$ ) serum creatinine levels (sCr) were excluded from the study $(n=66)$. The whole-body CT algorithm of the University Hospital Zurich was changed at the end of 2014. To avoid a potential study bias, we limited our analysis to the above-mentioned period. A total of 284 patients met inclusion criteria and were further analyzed.

\section{Setting}

Severely injured patients are admitted to the resuscitation area of our level I trauma center. In the division of trauma surgery, a standardized clinical approach is provided in the resuscitation area. The standard diagnostic algorithm includes a native head and neck $\mathrm{CT}$ followed by a primary contrast enhanced $(100 \mathrm{ml}$ iso-osmolar, non-ionic iodinated contrast material [ $300 \mathrm{mg}$ iodine per millilitre, iopromide (Ultravist $^{\circledR}$; Bayer Healthcare, Leverkusen, Germany)] followed by a saline flush of $30 \mathrm{ml}$ [19]) chest and abdomen $\mathrm{CT}$ during the whole study period. After initial stabilization in collaboration with the anesthesiologists, damage control or early total care surgery is performed [20], and patients 
are subsequently transferred to the intensive care unit for further treatment.

\section{Variables and data collection}

Demographics (age, gender comorbidities), injury severity score (ISS), complications, length of stay (LOS), need for hemodialysis, and death were extracted from the electronic medical record (KISIM, Cistec AG, Zürich, Switzerland). Extracted laboratory values included hemoglobin and fibrinogen level at admission (g/l), initial base excess (mmol/l) as well as serum lactate $(\mathrm{mmol} / \mathrm{l})$, initial and follow-up SCr levels. In addition, the following data were extracted from the anesthesia records until the admission to the ICU: volume of resuscitation fluids like crystalloid solution (in liters), the use of allogeneic blood products such as packed red blood cells (RBC, in numbers), fresh frozen plasma (FFP, yes/no), platelet concentrates (yes/no) and the use of fibrinogen (yes/no).

Contrast-induced nephropathy (CIN) was defined as a relative increase in serum creatinine $>25 \%$ of the baseline value or

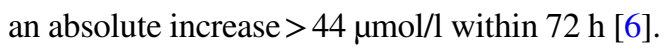

Complications were defined as the presence of acute delirium, myocardial infarction, deep vein thrombosis, pulmonary embolism, urinary tract infection, surgical site infection or complications such as pneumonia, sepsis, SIRS, cerebrovascular incidents or coagulopathy during the hospitalization [21].

\section{Study endpoints}

The main goal of this study was to determine prevalence of and to identify independent risk factors for CIN.

Additionally, we assessed the clinical impact of CIN on the need of hemodialysis, complications rates, length of stay and mortality.

\section{Statistical analyses}

Categorical data are reported as frequency and percent and numerical data as mean \pm standard deviation (SD). The Chisquare and Fisher's exact test were used to compare categorical data and the Mann-Whitney $U$-test for numerical data. Age, gender, Injury Severity Score for anatomic severity, and lactate level for physiologic severity were entered in multiple regression analysis as suggested by Haider et al. ("Bare minimum") [22]. In addition, CIN was forced into the models where applicable. Model fit was assessed using the Hosmer-Lemeshow test. The Glasgow Coma Scale and need for ventilator use were not used in these models since all patients were intubated at arrival. Lactate level was skewed to the right and therefore logarithmically transformed. All statistical analyses were performed by IBM SPSS Statistics 23 (IBM Corp., Armonk, NY, USA). Missing values were not entered into the analysis. A $p$ value of $<0.01$ was considered statistically significant, a $p$ value of 0.01-0.049 showed suggestive evidence [23].

\section{Results}

\section{Prevalence of and independent risk factors for CIN}

Prevalence of CIN was present in 41 out of 284 patients (14\%) (Table 1). Patients suffering from CIN had a significant lower hemoglobin level at admission $(p=0.002)$, were on average 9 years older $(p=0.019)$, had a slightly lower creatinine $(p=0.026)$ and lactate level $(p=0.036)$ at admission. Systolic blood pressure at admission was similar in both groups $(p=0.094)$. Patients with CIN received slightly more crystalloids $(p=0.042)$, fibrinogen $(p=0.027)$, and red blood cells $(p=0.017)$. In multivariate analysis, there

Table 1 Summary of patient characteristics between the CIN and no CIN group

\begin{tabular}{llll}
\hline & No CIN & CIN & $p$ value \\
& $n=243(86 \%)$ & $n=41(14 \%)$ & \\
\hline Age (years) & $46 \pm 22$ & $55 \pm 23$ & 0.019 \\
Gender (male) & $192(79 \%)$ & $29(71 \%)$ & 0.238 \\
Comorbidities & $145(60 \%)$ & $25(61 \%)$ & 0.875 \\
Blunt trauma & $235(97 \%)$ & $40(98 \%)$ & 0.673 \\
Injury Severity Score & $28 \pm 17$ & $30 \pm 16$ & 0.296 \\
AIS head & $3.1 \pm 1.8$ & $3.2 \pm 1.8$ & 0.733 \\
AIS face & $0.7 \pm 1.1$ & $0.6 \pm 1.2$ & 0.765 \\
AIS chest & $1.5 \pm 1.8$ & $1.6 \pm 1.7$ & 0.771 \\
AIS abdomen & $0.6 \pm 1.2$ & $0.7 \pm 1.3$ & 0.719 \\
AIS extremity & $1.2 \pm 1.4$ & $1.5 \pm 1.6$ & 0.316 \\
AIS integument & $0.9 \pm 0.9$ & $0.9 \pm 0.8$ & 0.961 \\
Heart rate at admission & $88 \pm 24$ & $86 \pm 22$ & 0.513 \\
$\quad$ bpm) & & & \\
Systolic blood pressure at & $121 \pm 28$ & $112 \pm 21$ & 0.094 \\
$\quad$ admission (mmHg) & & & \\
Serum creatinine ( $\mu$ mol/l) & $81 \pm 29$ & $73 \pm 26$ & 0.026 \\
Hemoglobin (g/l) & $11.7 \pm 2.4$ & $10.4 \pm 2.7$ & 0.002 \\
Fibrinogen (g/l) & $2.1 \pm 0.7$ & $2.2 \pm 1.1$ & 0.645 \\
Lactate level (mmol/l) & $2.7 \pm 2.6$ & $2.0 \pm 1.7$ & 0.036 \\
Base excess (mmol/l) & $-4.2 \pm 4.6$ & $-4.2 \pm 4.5$ & 0.800 \\
Crystalloid (l) & $2.2 \pm 2.9$ & $2.6 \pm 2.4$ & 0.042 \\
Red blood cells $(n)$ & $1.3 \pm 4.9$ & $2.3 \pm 4.7$ & 0.017 \\
Fresh frozen plasma & $13(5.4 \%)$ & $4(9.8 \%)$ & 0.287 \\
Platelets & $18(7.5 \%)$ & $7(17 \%)$ & 0.069 \\
Fibrinogen & $80(33 \%)$ & $21(51 \%)$ & 0.027 \\
Hemodialysis & $8(3.3 \%)$ & $6(15 \%)$ & 0.008 \\
Complications & $136(56 \%)$ & $29(71 \%)$ & 0.076 \\
Length of stay (days) & $14 \pm 13$ & $18 \pm 20$ & 0.407 \\
Mortality & $55(23 \%)$ & $13(32 \%)$ & 0.208 \\
\hline
\end{tabular}

CIN contrast-induced nephropathy 
Table 2 Risk factors for CIN

\begin{tabular}{lllll}
\hline & OR & $95 \%$ CI & & $p$ value \\
\hline Age (years) & 1.017 & 1.001 & 1.033 & 0.035 \\
Injury Severity Score & 1.008 & 0.988 & 1.029 & 0.430 \\
Gender (Male) & 0.806 & 0.370 & 1.760 & 0.589 \\
Lactate level (logarithmic) & 0.269 & 0.080 & 0.906 & 0.034 \\
\hline
\end{tabular}

Multivariable logistic regression analysis with following explanatory variables: age, gender, Injury Severity Score for anatomic severity, and logarithmically transformed lactate level for physiologic severity

$C I$ confidence interval, $O R$ odds ratio

is suggestive evidence that age $(p=0.035)$ and lactate level $(p=0.034)$ influenced the development of $\operatorname{CIN}\left(R^{2}=0.071\right)$ (Table 2).

\section{Hemodialysis}

Hemodialysis was necessary in 6 out of 41 CIN patients $(15 \%)$ and 8 out of 243 patients $(3.3 \%)$ without CIN $(p=0.008)$ (Table 1). The use of fresh frozen plasma $(p=0.006)$ and platelets $(p=0.004)$ were significantly, Injury Severity Score $(p=0.034)$, amount of infused crystalloids ( $p=0.012)$, numbers of RBC $(p=0.040)$ and use of fibrinogen $(p=0.023)$ were slightly associated with hemodialysis. The low numbers of hemodialysis did not allow multivariate analysis.

\section{Complications}

Complications during hospital stay occurred in 29 CIN patients $(71 \%)$ and in 136 patients $(56 \%)$ without CIN $(p=0.076)$. Pneumonia (10/29), wound infections $(4 / 29)$, deep vein thrombosis (4/29) and sepsis (3/29) were the most common complications in patients suffering from CIN. Injury Severity Score $(p<0.001)$, hemoglobin $(p=0.001)$ and fibrinogen at admission $(p=0.002)$, base excess $(p=0.001)$, amount of crystalloids $(p<0.001)$, numbers of RBC $(p=0.001)$, use of fibrinogen $(p<0.001)$ and FFP $(p=0.008)$ were significantly associated with the development of complications. However, preexisting comorbidities ( $p=0.024)$ were only slightly associated with the development of complications. In multivariate analysis, ISS was a risk factor for complications $(p=0.004)$, but not CIN $\left(R^{2}=0.083\right)($ Table 3$)$.

\section{Length of hospital stay}

The length of hospital stay was not affected by CIN $(18 \pm 20$ with vs. $14 \pm 13$ days without CIN, $p=0.407$ ) (Table 1 ).
Table 3 Risk factors for any complications

\begin{tabular}{lllll}
\hline & OR & $95 \%$ CI & & $p$ value \\
\hline CIN & 2.029 & 0.960 & 4.291 & 0.064 \\
Lactate level (logarithmic) & 2.027 & 0.867 & 4.737 & 0.103 \\
Injury Severity Score & 1.025 & 1.008 & 1.042 & $\mathbf{0 . 0 0 4}$ \\
Age (years) & 0.998 & 0.986 & 1.010 & 0.730 \\
Gender (male) & 0.939 & 0.511 & 1.727 & 0.840 \\
\hline
\end{tabular}

Bold values indicate significant values

Multivariable logistic regression analysis with following explanatory variables: age, gender, Injury Severity Score for anatomic severity, and logarithmically transformed lactate level for physiologic severity

$C I$ confidence interval, $C I N$ contrast-induced nephropathy, $O R$ odds ratio

\section{Mortality}

In-hospital mortality was $32 \%$ in patients with and $23 \%$ in patients without CIN $(p=0.208)$ (Table 1). Age $(p<0.001)$, comorbidities $(p<0.001)$, ISS $(p<0.001)$, hemoglobin at admission $(p<0.001)$, base excess $(p<0.001)$, amount of RBC $(p=0.006)$, use of FFP $(p=0.007)$ or platelets $(p<0.001)$ were significantly, lactate level $(p=0.044)$ slightly associated with death. CIN was not an independent risk factor for mortality $\left(R^{2}=0.435\right)$. However, age $(p<0.001)$ and a higher ISS $(p<0.001)$ were independent predictors for mortality (Table 4 ).

\section{Discussion}

Most of the research about CIN has been performed in patients undergoing percutaneous coronary interventions (PCI). To our knowledge, this study is one of few studies investigating CIN in the population of severe trauma and the only one in an European trauma center [24]. We thus intentionally focused on severely injured and already intubated patients. Due to our standardized diagnostic approach, they are most likely to undergo immediate contrast enhanced

Table 4 Risk factors for mortality

\begin{tabular}{llllr}
\hline & OR & $95 \%$ CI & \multicolumn{1}{r}{$p$ value } \\
\hline CIN & 1.192 & 0.480 & 2.962 & 0.706 \\
Injury Severity Score & 1.098 & 1.066 & 1.130 & $<\mathbf{0 . 0 0 1}$ \\
Lactate level (logarithmic) & 1.618 & 0.533 & 4.916 & 0.396 \\
Gender (male) & 1.051 & 0.446 & 2.480 & 0.909 \\
Age (years) & 1.030 & 1.013 & 1.047 & $\mathbf{< 0 . 0 0 1}$ \\
\hline
\end{tabular}

Bold values indicate significant values

Multivariable logistic regression analysis with following explanatory variables: age, gender, Injury Severity Score for anatomic severity, and logarithmically transformed lactate level for physiologic severity

$C I$ confidence interval, $C I N$ contrast-induced nephropathy, $O R$ odds ratio 
whole-body CT regardless of any pre-existing medical condition (e.g., renal insufficiency, diabetes mellitus), allergies or specific injuries. The prevalence of CIN was $14 \%$ in this population and we could identify lactate level as well as age as independent risk factors. Patients suffering from CIN were more likely to need hemodialysis (4.7 times), but the complication and mortality rate as well as length of hospital stay were not significantly affected by CIN.

The prevalence of CIN was $14 \%$ in our study population, which is slightly higher than described by other authors. Comparable studies found a prevalence ranging from 2.1 to $5.1 \%[3,4,13,25]$. Other studies in non-trauma patients reported a prevalence of $0.8-4.7 \%$ [26, 27]. The reason for our higher prevalence is probably due to our cohort compromising only severely injured patients. Age was a suggestive risk factor for CIN in our cohort. This is comprehensible and in accordance with other studies [28]. A lower lactate level was also a suggestive risk factor. This seems counterintuitive. However, the authors interpret this due to the increased need of volume and transfusions of these patients, as allogenic blood products are associated with a number of harmful side effects $[29,30]$. The question remains if an aggressive volume and transfusion management really increases the risk of CIN. Low hemoglobin level on admission was significantly associated with nephropathy, as also described by $\mathrm{Xu}$ and colleagues in patients undergoing coronary angioplasty or PCI [31]. Other authors did not find anemia to be a risk factor for $\mathrm{CIN}$, at least in cardiac patients $[32,33]$. Banda et al. found that anemia was not a risk factor for CIN, but CIN in combination with low hemoglobin levels doubled the mortality in their population [34]. Likewise, there is suggestive evidence that the amount of transfused red blood cell units is associated with CIN. There is possibly an exponentiating effect. Red blood cell transfusion of itself is a clear risk factor for acute kidney injury; in a meta-analysis by Karkouti et al., every unit of red blood cells increased the risk for acute kidney injury by $10-20 \%$ in cardiac surgery patients [35].

The development of nephropathy in polytraumatized patients is an intricate process. Vassiliu and his colleges analyzed trauma patients after angiographic embolization and found that age, hypovolemia, renal trauma and severe injuries were no risk factors for CIN in their population [36]. In contrast, Matsushima et al. found a higher ISS to be an independent risk factor for developing CIN [37]. The literature shows several strategies to prevent CIN. Because of the feasibility in this setting and the lack of doubtless proof [10] no routine administration of $\mathrm{n}$-acetylcysteine was performed in our hospital.

The hemodialysis rate was $15 \%$. Other studies found $0.3-7 \%$ of patients requiring hemodialysis after the administration of contrast agents $[4,38]$. The presence of CIN significantly increased the risk for hemodialysis in our population. One study found a higher rate of hemodialysis in patients developing CIN but was not able to identify CIN as a significant risk factor [39]. In our cohort, a higher ISS, an increased amount of crystalloids, allogeneic blood transfusion and procoagulant agents were associated with an increased need for hemodialysis. As with the nephropathy, the question remains about the causality.

Severely injured patients had a high complication rate, independent of CIN. Most of these complications were infections (pneumonia, cystitis) or wound complications (seroma, surgical site infection). The ISS was the most important risk factor for complications, which is consistent with the results of many other trauma studies. Patients suffering from CIN had not a higher risk of experiencing complication during their hospitalization.

Age and ISS influenced the mortality rate in our study, but not CIN. Other studies found an increased 1-year mortality in patients suffering from CIN after cardiac interventions. But it remains unclear if this due to CIN or the present underlying conditions and comorbidities, asking for the contrast-enhanced CT [40, 41].

There are some limitations to this study. Due to the retrospective design of our study we can only deduct association and not causation. There may be some confounders which we cannot detect and correct retrospectively. The whole-body CT algorithm of the University Hospital Zurich was changed at the end of 2014. To avoid a potential study bias, we limited our analysis to the period from 2008 to the end of 2014 . We intentionally focused on prehospital intubated patients due to our pre-defined, consistent diagnostic approach. All intubated trauma patients underwent immediately contrast-enhanced wholebody $\mathrm{CT}$ regardless of the injury pattern; the presence of a known renal impairment, allergies, or $\mathrm{SCr}$ levels was never known prior to the CT scan in these patients. Not intubated trauma patients may have undergone a different diagnostic approach due to the possibility to take a medical history at admission and to consider contraindication to perform a contrast enhanced whole-body CT scan. Available long-term data were incomplete and did not allow us to perform follow-up examinations to detect persistent renal failure or injury. Last, our analyzed patient cohort was not big enough to control for more than five factors in multivariate analysis.

We conclude that even in severely injured patients it is safe to perform contrast enhanced whole-body CT without further harming these patients. Even if CIN occurs, a benign course is likely and the patient's outcome, especially mortality and length of hospital stay are not affected. Further research is needed to investigate the effect of fluid and blood management on the development of CIN and the long-term outcome of patients affected by CIN. 
Authors contributions $\mathrm{AB}, \mathrm{AK}$ and $\mathrm{PS}$ contributed to data collection, data interpretation, drafting the manuscript and critical revision of the manuscript. KOJ, DRS, HPS and HCP contributed to data interpretation and critical revision of the manuscript. VN participated in the design and coordination of the study, contributed to statistical analysis, data collection and interpretation as well as drafting and critical revision of the manuscript. All authors read and approved the final manuscript.

Funding Open access funding provided by University of Zurich.

\section{Compliance with ethical standards}

Conflict of interest $\mathrm{AB}, \mathrm{KOJ}, \mathrm{BS}, \mathrm{HPS}, \mathrm{HCP}, \mathrm{VN}$ : none. AK: received honoraria for lecturing from Bayer AG (Zürich, Switzerland). PS: received honoraria for lecturing from Vifor Pharma (Munich, Germany). DS: Dr. Spahn's academic department is receiving grant support from the Swiss National Science Foundation, Berne, Switzerland, the Ministry of Health (Gesundheitsdirektion) of the Canton of Zurich, Switzerland for Highly Specialized Medicine, the Swiss Society of Anesthesiology and Reanimation (SGAR), Berne, Switzerland, the Swiss Foundation for Anesthesia Research, Zurich, Switzerland, CSL Behring, Berne, Switzerland, Vifor SA, Villars-sur-Glâne, Switzerland. Dr. Spahn is the co-chair of the ABC-Trauma Faculty, managed by Physicians World Europe GmbH, Mannheim, Germany and sponsored by unrestricted educational grants from Novo Nordisk Health Care AG, Zurich, Switzerland, CSL Behring GmbH, Marburg, Germany, LFB Biomédicaments, Courtaboeuf Cedex, France and Octapharma AG, Lachen, Switzerland. In the past 5 years, Dr. Spahn has received honoraria or travel support for consulting or lecturing from the following companies and organizations: Danube University of Krems, Austria, US Department of Defense, Washington, USA, European Society of Anesthesiology, Brussels, BE, Korea, Korean Society for Patient Blood Management, Seoul, Korea, Korean Society of Anesthesiologists, Seoul, Baxter AG, Volketswil, Switzerland, Baxter S.p.A., Roma, Italy, Bayer (Schweiz) AG, Zürich, Switzerland, Bayer Pharma AG, Berlin, Germany, B. Braun Melsungen AG, Melsungen, Germany, Boehringer Ingelheim (Schweiz) GmbH, Basel, Switzerland, BristolMyers-Squibb, Rueil-Malmaison Cedex, France and Baar, Switzerland, CSL Behring GmbH, Hattersheim am Main, Germany and Berne, Switzerland, Celgene International II Sàrl, Couvet, Switzerland, Curacyte AG, Munich, Germany, Daiichi Sankyo (Schweiz) AG, Thalwil, Switzerland, Fresenius SE, Bad Homburg v.d.H., Germany, GlaxoSmithKline GmbH \& Co. KG, Hamburg, Germany, Haemonetics, Braintree, MA, USA, LFB Biomédicaments, Courtaboeuf Cedex, France, Merck Sharp \& Dohme AG, Luzern, Switzerland, Octapharma AG, Lachen, Switzerland, Organon AG, Pfäffikon/SZ, Switzerland, PAION Deutschland GmbH, Aachen, Germany, Pharmacosmos A/S, Holbaek, Denmark, Photonics Healthcare B.V., Utrecht, Netherlands, ratiopharm Arzneimittel Vertriebs-GmbH, Vienna, Austria, Roche Diagnostics International Ltd, Reinach, Switzerland, Roche Pharma (Schweiz) AG, Reinach, Switzerland, Sarstedt AG \& Co., Sevelen, Switzerland and Nümbrecht, Germany Schering-Plough International, Inc., Kenilworth, New Jersey, USA, Tem International GmbH, Munich, Germany, Verum Diagnostica GmbH, Munich, Germany, Vifor Pharma, Munich, Germany, Vienna, Austria and Villars-sur-Glâne, Switzerland, Vifor (International) AG, St. Gallen.

Open Access This article is licensed under a Creative Commons Attribution 4.0 International License, which permits use, sharing, adaptation, distribution and reproduction in any medium or format, as long as you give appropriate credit to the original author(s) and the source, provide a link to the Creative Commons licence, and indicate if changes were made. The images or other third party material in this article are included in the article's Creative Commons licence, unless indicated otherwise in a credit line to the material. If material is not included in the article's Creative Commons licence and your intended use is not permitted by statutory regulation or exceeds the permitted use, you will need to obtain permission directly from the copyright holder. To view a copy of this licence, visit http://creativecommons.org/licenses/by/4.0/.

\section{References}

1. Feldkamp T, Kribben A. Contrast media induced nephropathy: definition, incidence, outcome, pathophysiology, risk factors and prevention. Minerva Med. 2009;99(2):177-96.

2. Chong E, Poh KK, Liang S, et al. Risk factors and clinical outcomes for contrast-induced nephropathy after percutaneous coronary intervention in patients with normal serum creatinine. Ann Acad Med Singapore. 2010;39(5):374-80.

3. Hipp A, Desai S, Lopez C, et al. The incidence of contrastinduced nephropathy in trauma patients. Eur J Emerg Med. 2008;15(3):134-9.

4. McGillicuddy EA, Schuster KM, Kaplan LJ, et al. Contrastinduced nephropathy in elderly trauma patients. The Journal of Trauma. 2010;68(2):294-7.

5. Nough H, Eghbal F, Soltani M, et al. Incidence and main determinants of contrast-induced nephropathy following coronary angiography or subsequent balloon angioplasty. CardioRenal Med. 2013;3(2):128-35.

6. Owen RJ, Hiremath S, Myers A, et al. Canadian Association of Radiologists consensus guidelines for the prevention of contrast-induced nephropathy: update 2012. Can Assoc Radiol J. 2014;65(2):96-105.

7. Haider M, Yessayan L, Venkat KK, et al. Incidence of contrastinduced nephropathy in kidney transplant recipients. Transpl Proc. 2015;47(2):379-83.

8. Chong E, Poh KK, Shen L, et al. Diabetic patients with normal baseline renal function are at increased risk of developing contrast-induced nephropathy post-percutaneous coronary intervention. Singapore Med J. 2009;50(3):250-4.

9. McCullough PA, Wolyn R, Rocher LL, et al. Acute renal failure after coronary intervention: incidence, risk factors, and relationship to mortality. Am J Med. 1997;103(5):368-75.

10. Tao SM, Wichmann JL, Schoepf UJ, et al. Contrast-induced nephropathy in CT: incidence, risk factors and strategies for prevention. Eur Radiol. 2016;26(9):3310-8. https://doi. org/10.1007/s00330-015-4155-8.

11. Huber-Wagner S, Lefering R, Qvick LM, et al. Effect of wholebody CT during trauma resuscitation on survival: a retrospective, multicentre study. Lancet. 2009;373(9673):1455-61.

12. Jiang L, Ma Y, Jiang S, et al. Comparison of whole-body computed tomography vs selective radiological imaging on outcomes in major trauma patients: a meta-analysis. Scandi J Trauma Resusc Emerg Med. 2014;22(1):54.

13. Colling KP, Irwin ED, Byrnes MC, et al. Computed tomography scans with intravenous contrast: low incidence of contrastinduced nephropathy in blunt trauma patients. J Trauma Acute Care Surg. 2014;77(2):226-30.

14. Finigan R, Pham J, Mendoza R, et al. Risk for contrastinduced nephropathy in elderly trauma patients. Am Surg. 2012;78(10):1114-7.

15. Kim DY, Kobayashi L, Costantini TW, et al. Is contrast exposure safe among the highest risk trauma patients? J Trauma Acute Care Surg. 2012;72(1):61-6 (discussion 66-67). 
16. Collaborative ST. Perioperative intravenous contrast administration and the incidence of acute kidney injury after major gastrointestinal surgery: prospective, multicentre cohort study. Br J Surg. 2020;107(8):1023-32.

17. Eisenach JC, Kheterpal S, Houle TT. Reporting of observational research in anesthesiology: the importance of the analysis plan. Anesthesiology. 2016;124(5):998-1000.

18. Elm EV, Altman DG, Egger M, et al. guidelines for reporting observational studies Strengthening the reporting of observational studies in epidemiology (STROBE) statement: guidelines for reporting observational studies. BMJ. 2007;335(October):19-22.

19. Gordic S, Alkadhi H, Hodel S, et al. Whole-body CT-based imaging algorithm for multiple trauma patients: radiation dose and time to diagnosis. Br J Radiol. 2015;88(1047):20140616.

20. Stein P, Kaserer A, Sprengel K, et al. Change of transfusion and treatment paradigm in major trauma patients. Anaesthesia. 2017;72(11):1317-26.

21. Neuhaus V, Swellengrebel CHJ, Bossen JKJ, et al. What are the factors influencing outcome among patients admitted to a hospital with a proximal humeral fracture? Clin Orthop Relat Res. 2013;471(5):1698-706.

22. Haider AH, Hashmi ZG, Zafar SN, et al. Developing best practices to study trauma outcomes in large databases: an evidence-based approach to determine the best mortality risk adjustment model. J Trauma Acute Care Surg. 2014;76(4):1061-9.

23. Benjamin DJ, Berger JO, Johannesson M, et al. Redefine statistical significance. Nat Hum Behav. 2018;2(1):6-10. https://doi. org/10.1038/s41562-017-0189-z.

24. Bashir AA, Kong V, Skinner D, et al. Contrast-induced nephropathy following CT scan for trauma is not rare and is associated with increased mortality in South African trauma patients. Eur J Trauma Emerg Surg. 2019;45(6):1129-35.

25. Kulvatunyou N, Rhee PM, Carter SN, et al. Defining incidence and outcome of contrast-induced nephropathy among trauma: is it overhyped? Am Surg. 2011;77(6):686-9.

26. Garfinkle MA, Stewart S, Basi R. Incidence of CT contrast agentinduced nephropathy: toward a more accurate estimation. Am J Roentgenol. 2015;204(6):1146-51.

27. Lencioni R, Fattori R, Morana G, et al. Contrast-induced nephropathy in patients undergoing computed tomography (CONNECT) — a clinical problem in daily practice? Acta Radiol (Stockholm, Sweden: 1987). 2010;51(7):741-50.
28. Toprak O, Cirit M, Yesil M, et al. Impact of diabetic and prediabetic state on development of contrast-induced nephropathy in patients with chronic kidney disease. Nephrol Dial Transpl. 2007;22(3):819-26.

29. Kaserer A, Rössler J, Slankamenac K, et al. Impact of allogeneic blood transfusions on clinical outcomes in severely burned patients. Burns. 2020;46(5):1083-90. https://doi.org/10.1016/j. burns.2020.11.005.

30. Spahn DR, Spahn GH, Stein P. Evidence base for restrictive transfusion triggers in high-risk patients. Transfus Med Hemother. 2015;42(2):110-4.

31. Xu J, Zhang M, Ni Y, et al. Impact of low hemoglobin on the development of contrast-induced nephropathy: a retrospective cohort study. Exp Ther Med. 2016;12(2):603-10.

32. Liu Y, Tan N, Zhou Y-L, et al. The contrast medium volume to estimated glomerular filtration rate ratio as a predictor of contrastinduced nephropathy after primary percutaneous coronary intervention. Int Urol Nephrol. 2012;44(1):221-9.

33. Toprak O. Risk markers for contrast-induced nephropathy. Am J Med Sci. 2007;334(4):283-90.

34. Banda J, Duarte R, Dickens C, et al. Risk factors and outcomes of contrast-induced nephropathy in hospitalised South Africans. S Afr Med J. 2016;106(7):699-703.

35. Karkouti K. Transfusion and risk of acute kidney injury in cardiac surgery. Br J Anaesth. 2012;109(Suppl 1):i29-38.

36. Vassiliu P, Sava J, Toutouzas KG, et al. Is contrast as bad as we think? Renal function after angiographic embolization of injured patients. J Am Coll Surg. 2002;194(2):142-6.

37. Matsushima K, Peng M, Schaefer EW, et al. Posttraumatic contrast-induced acute kidney injury: minimal consequences or significant threat? J Trauma. 2011;70(2):415-9 (discussion 419-420).

38. Levy EM, Viscoli CM, Horwitz RI. The effect of acute renal failure on mortality. A cohort analysis. JAMA. 1996;275(19):1489-94.

39. McDonald RJ, McDonald JS, Carter RE, et al. Intravenous contrast material exposure is not an independent risk factor for dialysis or mortality. Radiology. 2014;273(3):714-25.

40. Rudnick M, Feldman H. Contrast-induced nephropathy: what are the true clinical consequences? Clin J Am Soc Nephrol. 2008;3(1):263-72.

41. Wichmann JL, Katzberg RW, Litwin SE, et al. Contrast-induced nephropathy. Circulation. 2015;132:1931-6. 